\title{
AVALIAÇÃO DE CARACTERÍSTICAS DOS ÁCIDOS HÚMICOS DE RESÍDUOS DE ORIGEM URBANA: II. MÉTODOS TERMODEGRADATIVOS (PIRÓLISE ACOPLADA À CROMATOGRAFIA GASOSA / ESPECTROMETRIA DE MASSA)(1)
}

\author{
L. P. CANELLAS ${ }^{(2)}$, G. A. SANTOS(3), \\ A. A. MORAE $S^{(4)} \& V$. M. RUMJ ANEK(4)
}

\begin{abstract}
RESUMO
Este trabalho objetivou investigar as principais unidades estruturais de ácidos húmicos obtidos do composto de resíduos sólidos urbano (AH-CRSU) e lodo de estação de tratamento de esgoto (AH-LETE) por meio da pirólise acoplada à cromatografia gasosa / espectrometria de massas. Os ácidos húmicos extraídos do lodo da estação de tratamento de esgotos apresentaram maior quantidade de mono e diaril ésteres de massa mais elevada. Os n-alcanos presentes nos $\mathbf{A H}$ LETE revelaram-se de cadeia mais curta do que nos AH-CRSU. Foram incorporados na estrutura dos $\mathrm{AH}$ fragmentos de ftalatos, provavelmente em virtude da contaminação da matéria orgânica por plásticos. Os compostos aromáticos presentes nos AH-LETE originaram-se, principalmente, de derivados do naftaleno e derivados do benzeno com substituição por cadeias alifáticas maiores do que AH-CRSU. Os compostos nitrogenados presentes mostraram-se essencialmente heteroaromáticos e os oxigenados furanos substituídos originados da degradação de carboidratos. Os compostos oxiaromáticos predominantes foram fenóis substituídos.
\end{abstract}

Termos para indexação: matéria orgânica, substâncias húmicas, investigação estrutural.

\footnotetext{
(1) Parte da Tese de Doutorado em Ciência do Solo do primeiro autor, apresentada à Universidade Federal Rural do Rio de J aneiro em março de 1999. Recebido para publicação em junho de 1999 e aprovado em junho de 2000.

(2) Engenheiro Agrônomo. Departamento de Sol os. Universidade Federal Rural do Rio deJ aneiro- UFRRJ . CEP 23851-970 Seropédica (RJ).

(3) Professor do Departamento de Solos da UFRRJ . Bolsista do CNPq.

(4) Professor do Departamento de Química da UFRRJ .
} 


\title{
SUMMARY: EVALUATION OF CHARACTERISTICS OF HUMIC ACIDS FROM URBAN WASTE COMPOST: II. THERMODEGRADATIVE METHODS (PYROLYSIS - GAS CHROMATOGRAPHY / MASS SPECTROMETRY)
}

\begin{abstract}
This research was conducted to investigate the principal structural units from humic acids obtained from urban waste compost (AH-CRSU) and sewage sludge (AH-LETE) by pyrol ysis - gas chromatography / mass spectrometry. Thehumic acids from sewagesludge presented most mono and diaryl esters with higher mass. Then-al cans in AH-LETE showed a shorter chain than AH-CRSU. Phthal atefragments wereincorporated into thehumic acid structures, probably due to an organic matter contamination by plastics. The aromatic compounds in the AH-LETE originated mainly from naphtalene derivatives and benzene derivatives with alquil chain substitutives higher than AH-CRSU. The nitrogenated compounds wereessentially heteroaromatic and theoxigenated compounds weresubstituted furans originated from carbohydrate degradation. The oxiaromatic compounds were substituted phenols.
\end{abstract}

Index terms: organic matter, humic substances, structural investi gation.

\section{NTRODUÇÃO}

A natureza complexa, heteropolimérica e polidispersa dos ácidos húmicos $(\mathrm{AH})$ extraídos de resíduos orgânicos de origem urbana, observada por diferentes métodos, foi descrita na primeira parte deste trabal ho (Canellas et al., 2000).

Com relação às diferentes propriedades físicoquímicas, os métodos analíticos, espectroscópicos e microscopia eletrônica de varredura foram eficientes para caracterizar os materiais estudados. Entretanto, do ponto de vista estrutural, ou seja, das unidades estruturais que constituem os ácidos húmicos, foram obtidas somente informações genéricas.

Uma alternativa a essa limitação é a análise de ácidos húmicos por meio de técnicas termodegradativas, como a pirólise acoplada à cromatografia gasosa / espectrometria de massas (P-CG/EM) (Schulten, 1993). O experimento da pirólise consiste essencialmente em fragmentar os ácidos húmicos pela transferência rápida de energia térmica e analisar as características desses fragmentos separados pela cromatografia gasosa por espectrometria de massas.

Segundo Sainz-J imenez (1994), a P-CG/EM foi usada, pela primeira vez, em amostras de terras no estudo da superfície do deserto da Califórnia (USA), para analisar compostos orgânicos e identificar moléculas traçadoras de atividade biológica, num ensaio biológico preparatório para a prospecção do sol o de Marte (Simmonds et al., 1969). Desde então, a P-CG/EM tem sido uma ferramenta útil na identificação de unidades estruturais da matéria orgânica do solo (Hempfling \& Schulten, 1990; Schnitzer \& Schulten, 1995), desubstâncias húmicas de solos (Schulten \& Schnitzer, 1992) e da água (Abbt-Braun, et al., 1989), na avaliação da poluição dos solos (Leeuw et al., 1986 ) e na identificação de mudanças na matéria orgânica pelas práticas de manejo (Preston et al., 1994; Stucynsk, et al., 1997).

O objetivo deste trabalho foi identificar as principais unidades estruturais de ácidos húmicos extraídos de resíduos orgânicos de origem urbana por meio do uso da P-CG/EM.

\section{MATERIAL E MÉTODOS}

Os ácidos húmicos foram extraídos do composto de resíduo sól ido urbano produzido pela Companhia de Limpeza U rbana do Município do Rio de J aneiro (Comlurb) e de lodo da estação de tratamento de esgotos da Companhia E stadual de Águas e E sgoto (CEDAE) do Rio de J aneiro, conforme descrito na primeira parte deste trabalho.

A análise termodegradativa das unidades estruturais foi realizada por meio da pirólise acoplada à cromatografia gasosa / espectrometria de massas (P-CG/EM), utilizando amostras de $1 \mathrm{mg}$, colocadas em cubeta de quartzo e pirol isadas a $600^{\circ} \mathrm{C}$ por 5s, com piroprobo CDS 100 (Chemical Data Systems, Oxford) equi pado com filamento de platina. O pirolisador foi acoplado por uma interface a $150^{\circ} \mathrm{C}$ a um cromatógrafo a gás HP 5890 série II (Hewlet Packard, Oackland, CA). Os produtos da pirólise foram separados em coluna de sílica fundida da Supelco - SPB - 5 (30 m x 0,25 mm de diâmetro interno), usando injeção dividida (split 1/120) com temperatura de $2200^{\circ} \mathrm{C}$ e taxa de aquecimento da coluna de $50^{\circ} \mathrm{C}$ a $300^{\circ} \mathrm{C}$ a $5^{\circ} \mathrm{C}$ min-1 e, após, foram analisados por um detector quadrupolar Hewlett Packard 5970 B, com temperatura de interface de $220^{\circ} \mathrm{C}$, temperatura da fonte de íons a $250^{\circ} \mathrm{C}$, energia deionizaçãoa 70 eV egravação dos espectros de massa na faixa de $\mathrm{m} / \mathrm{z} 40$ até $\mathrm{m} / \mathrm{z} 600$. I dentificaram-se os 
compostos por meio de busca el etrônica na biblioteca (Nist) disponível no computador do aparelho. $\mathrm{Na}$ busca, comparou-se o espectro de massa obtido com os existentes na biblioteca com $80 \%$ de semel hança na relação massa / carga dos fragmentos $(\mathrm{m} / \mathrm{z})$ e na intensi dade dos fragmentos. Após a identificação, o composto foi submetido à análise de fragmentação para confirmar a identificação.

\section{RESULTADOS E DISCUSSÃO}

Na figura $1(a, b)$, são apresentados os cromatogramas dos produtos voláteis separados após a pirólise dos ácidos húmicos estudados. A identificação e a possível composição estrutural dos produtos foram realizadas por meio da análise do padrão de fragmentação por espectrometria de massas e comparação com banco de dados. A análise comparativa dos espectros de massas dos fragmentos obtidos com o espectro de uma substância-padrão apresenta algumas limitações, principalmente na identificação dos íons moleculares de razão massa:carga com valor elevado e, ou, ímpar. Entretanto, a técnica de P-CG/EM representa um avanço considerável em relação às outras técnicas, como, por exemplo, as de reação de oxidação degradativa de substâncias húmicas (Griffth \& Schnitzer, 1989), em que são observados os produtos derivados da degradação por reações químicas dessas macromoléculas. Os resultados encontrados por meio da análise por P-CG/EM dos ácidos húmicos estudados encontram-se no quadro 1.

$\mathrm{Na}$ região de $\mathrm{C}$ alquílicos, foram identificados compostos com longas cadeias alquílicas $(\mathrm{m} / \mathrm{z} 270$, $\mathrm{m} / \mathrm{z}$ 185), principalmente de 2-metil hexadecano (m/z 192). A intensidade destes sinais é maior nos AH-CRSU, o que está de acordo com a maior área integrada na região entre $\delta_{C} 20$ e $\delta_{C} 48$ observada por RMN ${ }^{13} \mathrm{C}-\mathrm{CP} / \mathrm{MAS}$ e pela espectroscopia de infravermel ho (Canellas et al., 2000). Os compostos com fragmentos de razão massa:carga maior são predominantes nos AH-LETE (m/z 541, m/z 549, $\mathrm{m} / \mathrm{z} 555, \mathrm{~m} / \mathrm{z} 556, \mathrm{~m} / \mathrm{z} 559, \mathrm{~m} / \mathrm{z}$ 563, m/z 578, m/z 590) e correspondem ao padrão de fragmentação de mono e diaril ésteres com cadeia alquílica longa. Resultados semel hantes foram encontrados por Leinweber et al. (1996), em sol os com adição recente de lodos da estação de tratamento de esgotos. Picos intensos atribuídos a ácidos graxos foram encontrados nos ácidos húmicos estudados.

Nos AH-CRSU, é mais intensa a presença dos ácidos octadecanóico (m/z 284), heptadecanóico ( $\mathrm{m} / \mathrm{z} 282$ ) e palmítico (m/z 256), enquanto, nos $\mathrm{AH}$ LETE, predominam ácido hexanóico (m/z 120) e limoneno ( $\mathrm{m} / \mathrm{z} 136)$, ácidos graxos de cadeia mais curta. A presença de ácidos graxos voláteis, segundo Keeling et al. (1994), é indicativo de al gum grau de anaerobiose nos estádios iniciais da compostagem dos resíduos. Outro importante indicativo de que os $\mathrm{AH}$ estudados estão numa fase relativamenteinicial de evolução é a presença, em grande proporção, de ácidos graxos esterificados. De acordo com Khan \& Schnitzer (1972), a tendência, com a maturação dos $\mathrm{AH}$, é o desaparecimento dos ácidos graxos e a permanência de alcanos na região de $C$ alquílicos.

Analisando os picos de fragmentos eas correspondentes intensidades relativas, reconhecidos pela busca el etrônica na biblioteca Nist, verificou-se, em ambos os AH estudados, a presença de ftalato de dioctila e ftalato de neobutila (sinal do picograma mais intenso em AH-CRSU). A presença de ftalatos em resíduos de origem urbana foi observada por Ziogou et al. (1986). A pesar deal guns microrganismos e plantas sintetizarem ftalatos, a principal fonteéa produção industrial de plásticos. Analisando a
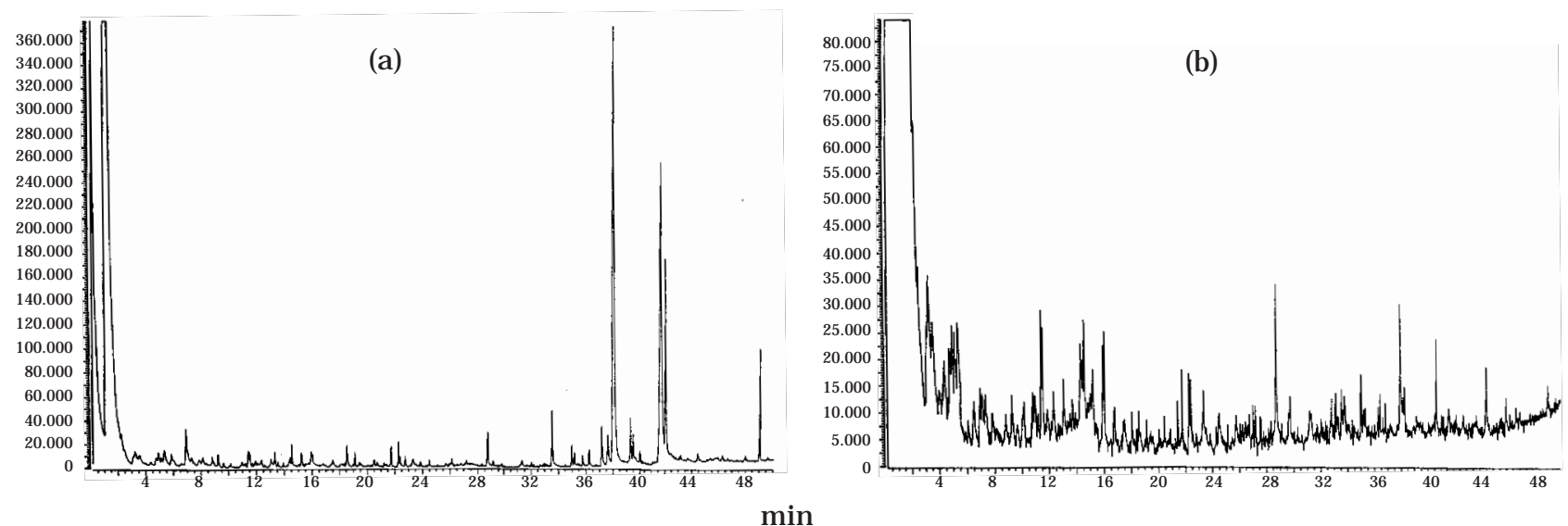

Figura 1. Cromatograma dos produtos da pirólise de áci dos húmicos extraídos do composto de resíduos sólidos (a) e de ácidos húmicos extraídos do lodo da estação de tratamento de esgotos (b). 
Quadro 1. Análise por pirólise - cromatografia gasosa/espectrometria de massas (p-cg/em) de ácidos húmi cos extraídos do composto de resíduo urbano (AH-CRSU) e de lodo da estação de tratamento de esgoto (AH-LETE)

\begin{tabular}{|c|c|c|c|c|c|}
\hline \multirow{2}{*}{ Pico } & \multirow{2}{*}{$\mathbf{T} \mathbf{R}$} & \multicolumn{2}{|c|}{ Ácido húmico } & \multirow{2}{*}{ Possível atribuição } & \multirow{2}{*}{$\begin{array}{l}\text { m/z (observado acima de } 50 \% \\
\text { de intensidade do espectro) }\end{array}$} \\
\hline & & LETE & $\overline{\text { CRSU }}$ & & \\
\hline 2 & 5,35 & (3) & (2) & metilbenzeno & $92,91,77$ \\
\hline$\overline{1}$ & 4,89 & (2) & (2) & monômeros de ligninas & $94,67,41,39,28$ \\
\hline 13 & 11,21 & (1) & (1) & dimetilpirrol & $95,60,39,27$ \\
\hline 3 & 5,87 & (1) & (2) & derivado do imidazol & $97,84,54,26$ \\
\hline 5 & 7,3 & (1) & (1) & 2,5-Furandiona & $98,54,26$ \\
\hline 27 & 15,91 & (4) & (4) & álcool furfurílico & $98,68,53,39$ \\
\hline 10 & 9,62 & (1) & (1) & heptanol & $98,68,40,27$ \\
\hline 8 & 8,8 & (1) & (1) & estireno & $104,78,77,51$ \\
\hline 7 & 8,13 & & (1) & xileno & $106,91,77,65$ \\
\hline 18 & 12,37 & (2) & (1) & pirrol 2-carboxialdeído & $95,94,66,39,28$ \\
\hline 19 & 12,57 & (1) & (1) & dimetil piridina & $107,77,67,27$ \\
\hline 4 & 6,89 & (1) & (3) & furfural & $98,96,67,39$ \\
\hline 9 & 9,23 & (1) & (1) & metilfenol & $108,107,93,80,54$ \\
\hline 6 & 7,84 & (1) & (1) & não identificado & $93,66,39$ \\
\hline 12 & 10,98 & (1) & (1) & diidroxibenzeno & $110,109,51,27$ \\
\hline 23 & 13,93 & (1) & (1) & 1,2-propadienil benzeno & $116,11542,27$ \\
\hline 16 & 11,72 & (1) & (1) & propenil 2-benzeno & $118,117,78,65$ \\
\hline 24 & 14,4 & (3) & (1) & ácido hexanóico & $116,105,73,60$ \\
\hline 25 & 14,53 & (3) & (3) & metil furfural & $120,107,90,77$ \\
\hline 26 & 15,22 & (2) & (2) & 4-metoxi fenol (quaiacol) & $124,109,95,81$ \\
\hline 22 & 13,55 & (1) & (1) & não identificado & $127,113,110,97,67,58,41,29$ \\
\hline 35 & 20,49 & (2) & (2) & ácido heptanóico & $130,115,73,60$ \\
\hline 28 & 16,02 & (3) & (2) & escatol & $132,99,56,28$ \\
\hline 14 & 11,42 & (2) & (1) & tetraidroquinolina & $133,94,66,39$ \\
\hline 15 & 11,52 & (2) & (1) & 2-metilbenzoxazol & $133,97,54,26$ \\
\hline 33 & 19,13 & (1) & 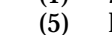 & hidroxiacetofenona & $120,91,51,27$ \\
\hline 21 & 13,32 & (1) & (2) & limoneno & $136,93,65,68,43$ \\
\hline 20 & 13,06 & (2) & (1) & derivado do naftaleno & $142,138,57,41$ \\
\hline 31 & 18,56 & (5) & (5) & cresol (metil guaiacol) & $138,122,94,95,77,55$ \\
\hline 30 & 18,38 & (2) & (2) & 1,4-dimetoxibenzeno & $138,123,95,77$ \\
\hline 32 & 19 & (1) & (1) & 3,5-diidroxi-2-metilpiranona & $126,98,97,109,98,69,41,29$ \\
\hline 38 & 21,75 & (5) & (5) & diidrohexose & $144,98,69,29$ \\
\hline 29 & 17,51 & (2) & (2) & não identificado & $148,122,115,107,91,85,73,60$ \\
\hline 45 & 24,5 & (2) & (2) & dimetilisopropilbenzeno & $148,105,57,43$ \\
\hline 41 & 22,75 & (1) & (2) & 1-(2-hidroxi-5-metilfenil)-etanona & $150,135,107,77$ \\
\hline 39 & 22,29 & (2) & (5) & 5-metil- 2-(1-metiletil)fenol & $150,135,107,77$ \\
\hline 37 & 21,23 & (1) & (1) & 4-etil- 2-metoxifenol & $152,137,122,91$ \\
\hline 42 & 23,27 & (2) & & 2,6-dimetóxi fenol & $154,139,111,65$ \\
\hline 44 & 23,88 & (1) & (1) & dimetil naftaleno & $160,73,60,29$ \\
\hline 46 & 26,15 & (1) & (3) & eugenol (alil quaiacol) & $164,149,121,77$ \\
\hline 40 & 22,39 & (5) & (1) & ácido 1, 2 -dicarboxílico benzeno & $166,148,104,76$ \\
\hline 47 & 27,3 & (2) & (1) & acetovanilona & $166,99,71,57,43$ \\
\hline 34 & 19,46 & (1) & (1) & ftalato de dioctila & $390,278,83,76$ \\
\hline 43 & 23,34 & (1) & (1) & ácido decanóico & $172,155,73,60$ \\
\hline 57 & 34,5 & (1) & & aldeído coniferílico & $178,150,71,57$ \\
\hline 55 & 32,93 & (1) & (1) & n-olefinas & $194,179,57,41$ \\
\hline 48 & 28,3 & (1) & (1) & álcool coniferílico & $180,137,94,77$ \\
\hline 50 & 29,1 & (1) & (1) & acetato de guaiacila & $180,165,137,91,65$ \\
\hline 52 & 32,01 & (1) & (1) & n-olefinas & $182,181,55,41$ \\
\hline 49 & 28,74 & (4) & (3) & n-alcano & $74,60,41,29$ \\
\hline 53 & 32,19 & (1) & (1) & 2-metilhexadecano & $240,192,99,43$ \\
\hline 36 & 20,72 & (1) & (1) & metoxipirocatecol & $140,125,97,51$ \\
\hline 56 & 33,55 & (5) & (2) & ácido tetradecanóico & $228,73,60,43$ \\
\hline 59 & 35,17 & (2) & (2) & metil dodecil benzeno & $242,73,55,41,29$ \\
\hline 63 & 37,61 & (3) & (3) & ácido hexadecanóico & $254,236,55,41$ \\
\hline 62 & 37,18 & (5) & (5) & ácido hexadecanóico ramificado & $256,73,60,43,29$ \\
\hline 64 & 38,13 & (5) & (5) & ácido palmítico & $256,129,60,43,29$ \\
\hline 65 & 39,29 & (1) & (1) & n-alcanos & $268,241,57,43,29$ \\
\hline 66 & 39,49 & (1) & (1) & n-alcanos & $268,241,57,41,29$ \\
\hline 67 & 40,01 & (1) & (1) & $\mathrm{n}$-alcanos & $270,256,43,57,43$ \\
\hline 61 & 36,25 & (3) & (2) & ftalato de neo-butila & $224,223,149,122,41$ \\
\hline 70 & 43,18 & & & não identificado & $280,256,236,67,55,29$ \\
\hline 17 & 11,93 & (1) & (1) & n-alcanos & $282,281,84,55,29$ \\
\hline 60 & 35,79 & (2) & (2) & n-alcanos & $282,281,83,73,43,29$ \\
\hline 68 & 41,71 & (1) & (1) & heptadeceno(8)-1-ácido carbônico & $282,264,83,69,55,29$ \\
\hline 69 & 42,07 & (1) & (1) & ácido octadecanóico & $284,241,83,73,43,29$ \\
\hline
\end{tabular}




\section{Quadro 1. Continuação}

\begin{tabular}{llll}
\hline Pico T R \begin{tabular}{l} 
Ácido húmico \\
\cline { 2 - 2 }
\end{tabular} & Possível atribuição & $\begin{array}{c}\mathrm{m} / \mathrm{z} \text { (observado acima de } 50 \% \\
\text { de intensidade do espectro) }\end{array}$
\end{tabular}

\begin{tabular}{|c|c|c|c|c|c|}
\hline 71 & 44,44 & & & alquil monoésteres e série homóloga/insaturado & $316,281,257,225,208,180,166,152,124,96,70,41,28$ \\
\hline 54 & 32,67 & & $(2)$ & derivados da-Galactose & $327,135,136,73,60$ \\
\hline 72 & 49,03 & & & ácidos graxos (C22) & $346,331,283,71,57,43$ \\
\hline 51 & 40,01 & & & n-ácidos graxos (C23) & $356,281,221,84,73,28$ \\
\hline 58 & 34,99 & $(2)$ & $(2)$ & Tocoferol & $430,242,73,43$ \\
\hline 73 & 6,13 & & & alquil monoésteres e série homóloga & $514,473,119,87,43$ \\
\hline 84 & 16,76 & & & alquil monoésteres e série homóloga & $542,504,452,90,51$ \\
\hline 75 & 25,93 & & & al cenos (C39) & $546,510,368,168$ \\
\hline 81 & 18,25 & $(2)$ & (1) & alquil monoésteres e série homóloga & $550,544,109,69,29$ \\
\hline 79 & 21,42 & $(2)$ & (1) & alquil monoésteres e série homóloga & $552,527,69,41$ \\
\hline 80 & 40,65 & (1) & (1) & alquil monoésteres e série homóloga & $554,508,105,71,57$ \\
\hline 83 & 14,17 & (1) & (2) & alquil monoésteres e série homóloga & $555,508,112,85,28$ \\
\hline 86 & 36,76 & (1) & (2) & alquil monoésteres e série homóloga & $556,481,110,97,41$ \\
\hline 76 & 46,25 & (1) & (2) & alcenos (C40) & $560,474,69,41$ \\
\hline 11 & 10,13 & (2) & $(2)$ & alquil monoésteres e série homóloga & $564,550,68,39$ \\
\hline 78 & 16,77 & (3) & (2) & alquil monoésteres e série homóloga & $578,180,117,92,41$ \\
\hline 77 & 14,3 & (2) & (2) & alquil monoésteres e série homóloga & $582,498,104,85,28$ \\
\hline 85 & 33,04 & (2) & (2) & alquil monoésteres e série homóloga & $590,511,469,439,387,355,318,265,203,186,130,93,38$ \\
\hline 82 & 24,49 & (2) & (2) & alquil monoésteres e série homóloga & $592,506,130,74,18$ \\
\hline 74 & 7,03 & (2) & (2) & alquil monoésteres e série homóloga & $598,551,108,80,41$ \\
\hline
\end{tabular}

(1) Intensidade no cromatograma entre 5-10\%. (2) $10-20 \%$. (3) $20-30 \% .{ }^{(4)} 30-50 \%$. ${ }^{(5)}$ Acima de $50 \%$.

decomposição de ftalatos nos solos, por meio da incubação de ácido ftálico, monobutilftalato e dibutilftalato marcados com ${ }^{14} \mathrm{C}$, I nman et al. (1984) observaram que a maior parte dos compostos é degradada até ${ }^{14} \mathrm{CO}_{2}$ como resultado da atividade microbiana que realiza a hidrólise do éster butílico equebra do anel aromático na posição $3,4 / 5,6$ ou 4,5 . $\mathrm{O}$ grupo carboxila é liberado como $\mathrm{CO}_{2}$ durante a quebra do anel ou depois de metabolizado (depois da degradação de succinato ou piruvato). A principal enzima responsável por essa atividade é a esterase que tem seu pleno funcionamento em condições de pH próximas à neutralidade. Parte do carbono dos ftalatos pode ser incorporada na biomassa microbiana e retida na matéria orgânica estável do solo. A identificação da incorporação de fragmentos deftalatos dedioctila na estrutura dos $\mathrm{AH}$ estudados, através de reações químicas ou enzimáticas, corrobora esta hipótese.

$\mathrm{Na}$ região de carbonos aromáticos, foram identificados, em maior quantidade, nos AH-LETE, os compostos derivados do naftaleno (dimetil naftal eno, $\mathrm{m} / \mathrm{z} 160$ ), derivados do benzeno (metildodedilbenzeno, $\mathrm{m} / \mathrm{z} 242$ ) e dimetilisopropilbenzeno ( $\mathrm{m} / \mathrm{z}$ 148). Os compostos derivados do benzeno presentes nos produtos da pirólise dos AH-CRSU apresentaram fragmentos de menor massa, tais como: $m$-xileno, tolueno $(\mathrm{m} / \mathrm{z} 93)$, propenil benzeno ( $\mathrm{m} / \mathrm{z} 117), 1,2-$ propadienilbenzeno (m/z 116). Estes compostos foram identificados também por Schnitzer \& Schulten (1992) como parte dos compostos aromáticos de ácidos húmi cos extraídos de solos de clima temperado. Uma série de compostos derivados da lignina foram identificados a partir da fragmentação dos produtos voláteis da pirólise dos ácidos húmicos, como os monômeros (m/z 108) e os compostos cresol (m/z 108), acetovanilona (m/z 166), álcool coniferílico (m/z 180), aldeído coniferílico (m/z 178) e tocoferol (m/z 429). Essa análise demonstra que, mesmo para matéria orgânica de origem urbana, a transformação de ligninas é importante mecanismo de formação de substâncias húmicas.

Comorepresentantes dos compostos oxiaromáticos, mais intensos nos AH-LETE, foram encontrados picos característicos de fenóis substituídos, principalmente 4-metoxifenol (m/z 124), 2-metoxi , 4 metilfenol (m/z 138), 4-etil-2-metoxifenol (m/z 152). Os AH-CRSU não apresentaram pico de íon molecular m/z 154 característico de 2,6 dimetoxifenol .

Nos carbonos oxigenados, foram observados furanos (m/z 107) substituídos, característicos da degradação de carboidratos, principalmente metilfurano al deí do (m/z 120), 2-metanol furano (m/ z 98), 2,5-furanodiona ( $\mathrm{m} / \mathrm{z}$ 98). NoAH-LETE, foram encontrados fragmentos constituintes de unidades de galactose (m/z 327) que não se encontravam presentes nos AH-CRSU.

A presença de compostos derivados das ligninas, de fenóis e de furanos na estrutura dos ácidos húmicos mostra que as vias de formação das substâncias húmicas em solos, descritas em Stevenson (1994), são válidas também para a evolução da matéria orgânica de resíduos de origem urbana. A alteração de compostos lignificados, as 
reações de condensação de polifenóis ea neossíntese microbiana de compostos são as principais vias de formação dessas substâncias húmicas.

Os picos obtidos da piról ise de grupos nitrogenados revelaram a predominância de compostos nitrogenados heterocícl icos nas unidades estruturais dos ácidos húmicos, principalmente os derivados do imi dazol ( $\mathrm{m} / \mathrm{z}$ 97), pirrol ( $\mathrm{m} / \mathrm{z}$ 94), pirróis substituídos (2,3 ou 2,4 ou 2,5 dimetil pirrol m/z 95), 1-H- pirrol 2carboxialdeído (m/z 107), tetraidroquinolina ( $\mathrm{m} / \mathrm{z} 133)$, 2-metilbenzoxazol $\left(\mathrm{C}_{8} \mathrm{H}_{7} \mathrm{NO}, \mathrm{m} / \mathrm{z} 133\right)$ e piridinas substituídas (dimetilpiridina, m/z 107). Apesar da análise de RMN ${ }^{13} \mathrm{C}$ indicar a presença de $\mathrm{N}$ aminoácidos, a identificação de $\mathrm{N}$-heterocíclico na estrutura dos ácidos húmicos, aliada à presença de ácidos graxos de cadeia al quílica longa e esterificados, mostra também a pouca transformação química desses $\mathrm{AH}$. Estes compostos representam uma reserva importantedenitrogênio, quando adicionados ao solo, pois sua transformação em $\mathrm{N}$-mineral disponível às plantas épossível com a transformação química dos $\mathrm{AH}$.

Foram encontrados também na estrutura dos $\mathrm{AH}$ estudados ácidos benzocarboxílicos (ácido 1,2benzodicarboxílico, m/z 166) e pico de maior intensidade nos AH-LETE de bis metil-propil ácido 1,2 benzocarboxílico. Ambos os ácidos apresentaram função cetona 1- (2-hidróxi-5-metilfenil) etanona $(\mathrm{m} / \mathrm{z}$ 150). A presença desses grupamentos pode ser uma evidência que permitejustificar a mai or acidez determinada nos AH-LETE ea maior área integrada na região de $\delta_{C}$ 165-180 ppm na espectroscopia de RMN ${ }^{13} \mathrm{C}-\mathrm{CP} / \mathrm{MAS}$.

\section{CONCLUSÕES}

1. Os ácidos húmi cos extraídos do lodo da estação de tratamento de esgotos apresentaram na composição dos produtos voláteis resultante da pirólise maior quantidade de mono e diaril ésteres de massa mais elevada. A presença de ácidos graxos na estrutura dos $\mathrm{AH}$ deveu-se ao possível estádio inicial de evol ução dessas substâncias. Os n-al canos presentes na estrutura dos AH-LETE foram de cadeia mais curta do que os presentes nos $\mathrm{AH}-\mathrm{CRSU}$.

2. Foram incorporados na estrutura dos $A H$ fragmentos de ftalatos de dioctila, provavelmente pela contaminação da matéria orgânica pelos plásticos existentes na matéria-prima dos resíduos.

3. Os compostos aromáticos presentes nos AHLETE originaram-se, principalmente, de derivados donaftaleno edobenzeno com substituição por cadeias al ifáticas maiores do que no AH-CRSU. Os compostos nitrogenados presentes mostravam-seessencial mente heteroaromáticos eos oxigenados furanos substituídos originados pela degradação de carboidratos. Os compostos oxiaromáticos predominantes foram os fenóis substituídos.

\section{AGRADECIMENTOS}

Os autores agradecem aos professores Raimundo Braz Filho e Ary Carlos Xavier Velloso, da Universidade Estadual do Norte Fluminense (UENF), pela revisão do texto.

\section{LITE RATURA CITADA}

ABBT-BRAUN, G. ; FRIMMEL, F.H. \& SCHULTEN, H.-R. Structural investigations of aquatic humic substances by pyrolysis-field ionization mass spectrometry and pyrolysisgas chromatography/mass spectrometry. Water Res., 23:1579-1591, 1989.

CANELLAS, L.P.; SANTOS, G.A.; MORAES, A.A.; RUMJ ANEK, V. \& OLIVARES, F.L. Avaliação de características deácidos húmicos extraídos de resíduos de origem urbana I: métodos espectroscópicos (Uv-Vis, IV, RMN 13C-CP/MAS) e microscopia eletrônica de varredura. R. Bras. Ci. Solo, 24:741-750, 2000.

GRIFFITH, S.M. \& SCHNITZER, M. Oxidative degradation of soil humic substances. In: HAYES, M.H.B.; MACCARTHY, P.; MALCOLM, R.L. \& SWIFT, R.S., eds. Humic substances II. New York, J ohn Wiley, 1989. p.70-98.

HEMPFLING, R. \& SCHULTEN, H.R. Chemical characterization of organic matter in forest soil by curie point pyrolysis CG-MS and pyrolysis-field ionization mass spectrometry. Org. Geochem., 15:131-145, 1990.

INMAM, J .C.; STRACHAN, S.D.; SOMMERS, L. \& NELSON, D.W. The decomposition of phthalate esters in soil. J. Environ. Sci. Health, 19:245-257, 1984.

KEELING, A.A.; MULLET, J .A.J . \& PATON, I.K. GC-Mass spectrometry of refuse-derived composts. Soil Biol. Biochem., 26:773-776, 1994.

KHAN, S.U. \& SCHNITZER, M. The retention of hidrophobic organic compounds by humic acid. Geochim. et Cosmochim. Acta, 36:745-754, 1972.

LEINWEBER, P.; BLUMENSTEIN, O. \& SCHULTEN, H.-R. Organic matter composition in sewage sludge farm soilsinvestigations by ${ }^{13} \mathrm{C}-\mathrm{nmr}$ and pyrolysis-field ionization mass spectrometry. Eur. J . Soil Sci., 47:71-80, 1996.

LEEUW, J.W.; LEER, E.W.B.; SINNINGHE DAMSLÉ, J . \& SHULYL, P.J.W. Screening of anthropogenic compounds in polluted sediments and soil by flash evaporation/ pyrolysis gas chromatography-mass spectrometry. Anal. Chem,. 58:1852-1857, 1986.

PRESTON, C.M.; HEMPLING, R.; SCHULTEN, H.-R., SCHNITZER, M.; TROFYMOW, J .A. \& AYELSON, D.E. Characterization of organic matter in a forest soil of coastal British Columbia by NMR and pyrolysis-field ionization mass spectrometry. Plant Soil, 158:69-82, 1994.

SAIZ-JIMENEZ, C. Application of pyrolysis - gas chromatography/mass spectrometry to soil chemistry. In: WORLD CONGRESS OF SOIL SCIENCE, 15., Acapulco, 1994. Symposia. Acapulco, 1994. v.3. p.65-84. 
SCHNITZER, M. \& PRESTON, C. Analysis of humic acids by solution and solid-state carbon-13 nuclear magnetic resonance. Soil Sci. Am. J ., 50:3226-3331, 1986.

SCHNITZER, M. \& SCHULTEN, H-R. Analysis of organic matter in soil extracts and whole soils by pyrolysis-mass spectrometry. Adv. Agron., 55:167-217, 1995.

SCHULTEN, H-R. \& SCHNITZER, M. Structural studies on soil humic acids by curie-point pyrolysis-gas chromatography/ mass spectrometry. Soil Sci., 153:205-224, 1992.

SCHULTEN, H-R. Analytical pyrolysis of humic substances and soils: geochemical, agricultural and ecological consequences. J . Anal. Appl. Pyrolysis, 25:97-122, 1993.
SIMMONDS, P.G.; SHULMAM, G.P. \& STEMBRIDGE, R. Organic analysis by pyrolysis - gas chromatography/mass spectrometry: a candidate experiment for the biological exploration of Mars. J . Chromatogr. Sci., 7:36-41, 1969

STEVENSON, J .F. Humus chemistry, genesis, composition, reactions. New york, J ohn Wiley, 1994. 443p.

STUCZYNSK, T.I.; MCCARTY, G.W.; REEVES, J .B. \& WRICH, $J$ J.B. Use of pyrolysis GC/MS for assessing changes in soil organic matter quality. Soil Sci., 162:97-105, 1997.

ZIOGOU, K.; KIRK, P.W.W. \& LESTER, J .N. Behaviour of phthalic acid esters during batch anaerobic digestion of sludge. Water Res., 23:743-748, 1986. 
L. P. CANELLAS et al. 